1. Rindi, G. \& Wiedenmann, B. Neuroendocrine neoplasia goes molecular - time for a change. Nat. Rev. Clin. Oncol. 16, 149-150 (2018)

2. Liu, E. et al. Assessment of NETest clinical utility in a US registry-based study. Oncologist https://doi.org/10.1634/theoncologist.2017-0623 (2018).

3. de Mestier, L. et al. Evaluating digestive neuroendocrine tumor progression and therapeutic responses in the era of targeted therapies: state of the art. Endocr. Relat. Cancer. 21, R105-R120 (2014).

4. Modlin, I. M. et al. The NETest: the clinical utility of multigene blood analysis in the diagnosis and management of neuroendocrine tumors. Endocrinol. Metab. Clin. North Am. 47, 485-504 (2018).

Tang, L. H. et al. Objective quantification of the Ki67 proliferative index in neuroendocrine tumors of the gastroenteropancreatic system: a comparison of digital image analysis with manual methods. Am. J. Surg. Pathol. 36, 1761-1770 (2012).
6. Bossuyt, P. M. et al. The STARD statement for reporting studies of diagnostic accuracy: explanation and elaboration. Ann. Intern. Med. 138, W1-W12 (2003).

7. Marotta, V. et al. Chromogranin A as circulating marker for diagnosis and management of neuroendocrine neoplasms: more flaws than fame. Endocr. Relat. Cancer. 25, R11-R29 (2018).

8. Capdevila, J. et al. Translational research in neuroendocrine tumors: pitfalls and opportunities. Oncogene 36, 1899-1907 (2017).

9. Strosberg, J. et al. United States-based practice patterns and resource utilization in advanced neuroendocrine tumor treatment. World J. Gastroenterol. 19, 2348-2354 (2013).

10. Sparano, J. A. et al. Adjuvant chemotherapy guided by a 21 -gene expression assay in breast cancer. N. Engl. J. Med. 379, 111-121 (2018)

Competing interests

The authors declare no competing interests.

\title{
Reply to 'Time for a change and to adopt a novel molecular genomic approach in NETs'
}

\section{Guido Rindi(D) and Bertram Wiedenmann}

We wish to respond to the letter from Lisa Bodei and collaborators (Bodei, L. et al. Time for a change and to adopt a novel molecular genomic approach in NETs. Nat. Rev. Clin. Oncol. https://doi.org/10.1038/s41571-0190185-5 (2019) $)^{1}$ in which they comment on our News \& Views piece (Rindi, G. \& Wiedenmann, B. Neuroendocrine neoplasia goes molecular - time for a change. Nat. Rev. Clin. Oncol. 16, 149-150 (2018)) $)^{2}$ on their article describing the prospective evaluation of the NETest in a cohort of 100 patients ${ }^{3}$. In their letter, Bodei et al. quote “.... an NIHfunded study evaluating the gene expression profiles of $>10,000$ tumours from The Cancer Genome Atlas (TCGA) database..."1 and cite their study ${ }^{3}$ to support this statement, but they ultimately fail to provide the claimed data. We wish to highlight that we were asked to scrutinize and comment on the data provided by Liu et al. ${ }^{3}$ and not the entire set of data generated by the use of the NETest. We feel that this premise is indicative of how Bodei and collaborators interpreted our commentary.

In their letter, Bodei et al. list five "factual inaccuracies" ${ }^{\prime 1}$ of our News \& Views article that we wish to clarify. First, according to the correspondents, we claimed that the Response Evaluation Criteria in Solid Tumors (RECIST) always enable an accurate assessment of the progression of neuroendocrine tumours (NETs). No such statement was made ${ }^{2}$, and neither were we asked to express our opinion on the efficacy of RECIST in clinical practice. However, it is factually accurate that they used RECIST in their study ${ }^{3}$ to evaluate tumour progression and compare with the results obtained with the NETest.

Second, Bodei et al. seem to imply that we recommended their data should be viewed with caution owing to intra-patient tumour heterogeneity. Given the high degree of heterogeneity of neuroendocrine neoplasia, however, we focused only on inter-patient heterogeneity and indeed we suggested that the application of this test would be challenged in larger patient cohorts stratified by site, tumour type, grade and/or stage ${ }^{2}$. Inter-patient tumour heterogeneity remains a crucial issue at different levels (clinical, genetic and circulating markers, including RNA and DNA). Blood-based tests do not necessarily reflect the same relative proportions of tumour-related RNA in the bloodstream as might be present in tumour cells owing to the occurrence of tumour cell anoikis or differentiation and to differences in cell volume. Their statement "Using liquid biopsy approaches, however, all clonal components are sampled" ${ }^{1}$ is currently unrealistic . $^{4}$ Furthermore, no evidence is available to indicate whether blood and tissue samples are subject to the same sampling limitations - indeed, cancer is a dynamic entity in which the levels of both RNA transcripts and protein expression change over time. Finally, the NETest does not encompass all the possible alterations in NETs, thus limiting its potential for general application ${ }^{3}$.

The third factual inaccuracy that Bodei et al. found in our News \& Views was the statement that they did not provide sensitivity data in their study ${ }^{3}$. Indeed, the data presented in the study do not enable robust calculations of the sensitivity of the NETest, including negative and positive predictive values, and none of these data was openly displayed.

Fourth, they comment that our statement that patient management depends on a multidisciplinary team (MDT) approach should be applied to all cancer types and not only to patients with NETs. We agree and insist that MDTs should continue to be considered as a mainstay of the management of these patients.

Finally, Bodei and collaborators attribute to us the statement that the cost-benefit ratio of the NETest is unfavourable. No such statement is present in our News \& Views ${ }^{2}$; rather, we expressed our general opinion about all blood-based molecular tests. Regardless, in the European setting of universal health-care coverage, the cost of such assays does, unfortunately, remain an issue.

In our opinion, the critical interpretation by Bodei et al. of our commentary is factually misplaced. Unfortunately, and for reasons we do not understand, Bodei et al. failed to appreciate the overall positive vision we provided about molecular testing in neuroendocrine cancer, both upfront in the title and in the good-news statement conclusion of our commentary ${ }^{2}$. We also certainly stressed the need for better work to understand the efficacy and limitations of such tests in clinical practice, but measurable advances will only be achieved when the dialogue between science and medicine is preserved.

Guido Rindi $\mathbb{D}^{1 *}$ and Bertram Wiedenmann ${ }^{2}$

'Fondazione Policlinico Universitario A. Gemelli IRCCS-Università Cattolica del Sacro Cuore, Rome, Italy.

${ }^{2}$ Charité, Campus Virchow Klinikum and Charité Mitte, University Medicine Berlin, Berlin, Germany. *e-mail: guido.rindi@unicatt.it https://doi.org/10.1038/s41571-019-0186-4

1. Bodei, L. et al. Time for a change and to adopt a novel molecular genomic approach in NETs. Nat. Rev. Clin. Oncol, https://doi.org/10.1038/s41571-019-0185-5 (2019).

2. Rindi, G. \& Wiedenmann, B. Neuroendocrine neoplasia goes molecular - time for a change. Nat. Rev. Clin. Oncol. 16, 149-150 (2018).

3. Liu, E. et al. Assessment of NETest Clinical utility in a U. S. registry-based study. Oncologist https://doi. org/10.1634/theoncologist.2017-0623 (2018). 4. Bardelli, A. \& Pantel, K. Liquid biopsies, what we do not know (yet). Cancer Cell 31, 172-179 (2017).

\section{Competing interests}

G.R. has received honoraria from Ipsen and Novartis for his role in their speaker's bureau. B.W. declares no competing interests. 